\title{
Salmonella enteritidis Infection Complicated by Acute Myocarditis: A Case Report and Review of the Literature
}

\author{
Panagiotis Papamichalis, ${ }^{1}$ Katerina Argyraki, ${ }^{1}$ Michail Papamichalis, ${ }^{2}$ \\ Argyris Loukopoulos, ${ }^{1}$ Georgios N. Dalekos, ${ }^{1}$ and Eirini I. Rigopoulou ${ }^{1}$ \\ ${ }^{1}$ Department of Medicine, Medical School, University of Thessaly, Biopolis, 41110 Larissa, Greece \\ ${ }^{2}$ Department of Cardiology, Medical School, University of Thessaly, Biopolis, 41110 Larissa, Greece
}

Correspondence should be addressed to Eirini I. Rigopoulou, eirigopoulou@med.uth.gr

Received 18 February 2011; Accepted 28 March 2011

Academic Editor: Jalal K. Ghali

Copyright (c) 2011 Panagiotis Papamichalis et al. This is an open access article distributed under the Creative Commons Attribution License, which permits unrestricted use, distribution, and reproduction in any medium, provided the original work is properly cited.

\begin{abstract}
Salmonella spp. is the cause of commonly encountered infections, with seasonal pattern of occurrence and worldwide distribution. Some of the clinical manifestations such as gastroenteritis and bacteremia are common, whereas others like mycotic aneurysms and osteomyelitis are infrequent especially in immunocompetent patients. Salmonella has been rarely described as a cause of myocarditis in the literature. We describe a case of an 18-year-old previously healthy male patient with myocarditis after Salmonella enteritidis infection. Clinical manifestations and diagnostic approach of this severe complication are discussed with a review of the literature.
\end{abstract}

\section{Introduction}

Myocarditis, clinically defined as inflammation of the myocardium, has a broad spectrum of causes including infectious, immune-mediated, and toxic [1]. The role of viruses as etiological factors, especially coxsackievirus and adenovirus, has been thoroughly investigated over the years [1]. The role of bacterial infections is obscure, as these infections are thought to be less commonly associated with myocarditis [1]. Nontyphoidal Salmonella gastroenteritis is a foodborne infection, which is usually self-limited. Bacteremia or other invasive complications are rare in immunocompetent persons.

We present a previously healthy male patient with acute myocarditis following Salmonella enteritidis infection. We also discuss the role of biomarkers of cardiac injury, ECG, echocardiogram, and endomyocardial biopsy in the diagnosis of myocarditis, in cases that follow infection with Salmonella spp.

\section{Case Report}

A 18-year-old previously healthy male presented to the emergency department (ED) of our hospital, with a 48-hour history of $>20 /$ day profuse, watery, nonbloody diarrheas, 5 times of vomiting, generalized abdominal, pain and fever up to $40^{\circ} \mathrm{C}$. His previous medical record was clear from coronary artery risk factors or any other pathology. On the same day and before attending our ED, he had visited another hospital. No laboratory studies were performed there, and after administration of i.v. liquid and electrolyte replacement, he was discharged with the diagnosis of gastroenteritis. Of relevance, 2 days before the onset of symptoms, the patient had eaten fried rice with egg and chicken in a Chinese restaurant.

Physical examination on admission to the hospital revealed temperature $40.1^{\circ} \mathrm{C}$, blood pressure $110 / 80 \mathrm{mmHg}$, heart rate 100 per minute, and respiratory rate 25 per minute. Cardiac examination was normal. Abdominal examination demonstrated generalized tenderness.

Laboratory tests performed in our ED showed increased leucocytes (14400) with neutrophilia (NE: 12400, LY: 700, MO: 1300), hemoglobulin $16.6 \mathrm{~g} / \mathrm{dL} /$ hematocrit $48.7 \%$, sodium $138 \mathrm{mmol} / \mathrm{L}$, and potassium $4.21 \mathrm{mmol} / \mathrm{L}$ with normal urea and creatinine levels $(26 \mathrm{mg} / \mathrm{dL}$ and $1.1 \mathrm{mg} / \mathrm{dL}$, resp.) and elevated C-reactive protein $(11.28 \mathrm{mg} / \mathrm{dL}$ with upper limit normal (ULN) 10). Creatine phosphokinase 


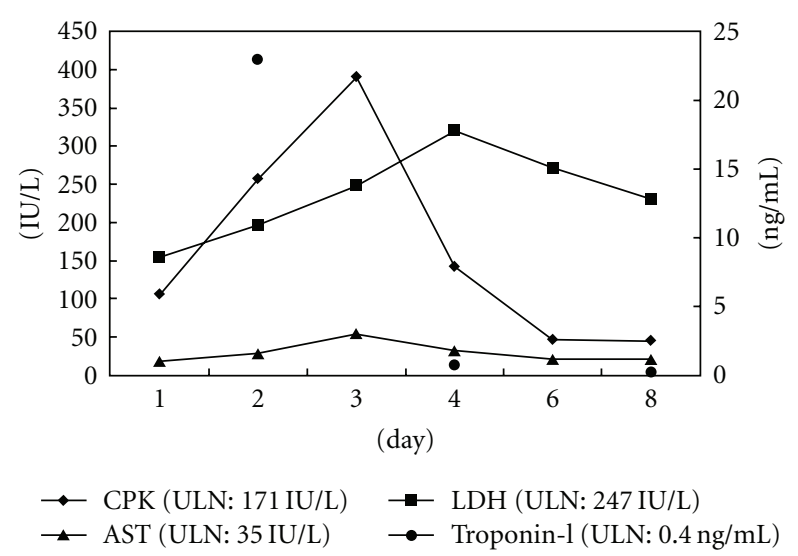

FIGURE 1: Laboratory results of cardiac biomarkers during hospitalization in a patient with Salmonella enteritidis associated acute myocarditis.

(CK): $107 \mathrm{U} / \mathrm{L}$ (ULN: 171), aspartate aminotransferase (AST): 19 IU/L (ULN: 35), and lactate dehydrogenase (LDH): 155 IU/L (ULN: 247) were normal (Figure 1). The electrocardiogram (ECG) performed in the ED was also normal, exhibiting upslope displacement of ST segment in precordial leads due to early repolarization (changes without clinical significance in young adults) (Figure 2(a)).

Admittance to our department was decided on the basis of clinical markers of dehydration, related to increased number and volume of diarrheas, and considering that the patient had sought medical help twice during a short period of time, indicating possibly the severity of symptoms or other more obscure and severe underlying pathology. Replacement of i.v. fluids $(0.9 \% \mathrm{NaCl})$ and electrolyte loses was initiated with subsequent improvement in the patient's clinical condition.

After 24 hours of hospitalization, the patient complained of chest discomfort for the duration of 30 minutes, with no pain reflection, no pain relief with any changes in body position, and absence of pleuritic pain characteristics. The ECG during that episode was without any changes from the one on admission. Troponin-I (TnI) (qualitative assay) was normal and symptoms resolved.

Few hours later, laboratory tests revealed slight elevation of CK (258 U/L), while AST and LDH levels were within normal limits (29 IU/L and $197 \mathrm{IU} / \mathrm{L}$, resp.) (Figure 1). A new ECG did not reveal changes compared to the previous ones, while an echocardiogram (ECHO) was also performed. It was normal without hypokinetic areas. The left ventricular ejection fraction was $70 \%$, and there was no pericardial fluid. TnI test was positive (qualitative assay) at that time, and assessment with a quantitative assay showed remarkable elevation of TnI (23 ng/mL; ULN: 0.4) (Figure 1).

Consequently, the patient was transferred to the coronary care unit for cardiac monitoring. AST, CK, and LDH reached maximum values on the third day of hospitalization ( $55 \mathrm{IU} / \mathrm{L}, 392 \mathrm{U} / \mathrm{L}$, and $249 \mathrm{IU} / \mathrm{L}$, resp.). AST and CK alongside with Tnl became normal on the fourth date and LDH on the sixth day (Figure 1). The ECG evolved with $\mathrm{T}$ wave inversion in lead III and biphasic $\mathrm{T}$ wave in leads $\mathrm{V}_{4}$ and $\mathrm{V}_{5}$ on the third day (Figure 2(b)). Abnormal Q waves were not observed at any timepoint. ECG normalized on the sixth day before his discharge from the hospital.

Myocardial infarction was ruled out based on the lack of coronary artery risk factors and the age of our patient, and the diagnosis of acute myocarditis was established. On the third day of admission and after diarrhea and fever had resolved, Salmonella enteritidis was isolated from the stool culture. Tests for other infectious causes of myocarditis were negative. Considering that clinical manifestations of heart involvement occurred simultaneously with those of gastroenteritis, and no other possible causes (drugs and autoimmune diseases) could be recognized, Salmonella was identified as the causative agent of acute myocarditis in this patient.

Our patient remained asymptomatic with no relapse of symptoms. Additionally to fluids and electrolytes, ramipril at low dose (2.5 mg per day) and acetylsalicylic acid (100 mg per day) were administrated and were continued following his discharge from the hospital. ECHO was repeatedly normal with identical features to those described above. ECG and biomarkers of cardiac injury (CK and $\mathrm{TnI}$ ) became normal as reported above. He was discharged after six days of hospitalization with followup from the cardiology outpatient clinic of our hospital. Nine months later (up to the time this paper was written), he remained in an excellent condition and with no indications of evolution to dilated cardiomyopathy.

\section{Discussion}

Myocarditis presents with a broad spectrum of clinical manifestations ranging from mild chest pain to cardiogenic shock and death [1]. Apart from the primary risks of developing fulminant heart failure or arrhythmias, dilated cardiomyopathy and chronic heart failure is the most dreadful and devastating long-term complication of myocarditis [1]. In a large single center, prospective study myocarditis accounted for $9 \%$ of cases of dilated cardiomyopathy [16]. Bacterial myocarditis is considered less frequent than myocarditis following viral infections and usually involves immunocompromised hosts or cases of sepsis with concomitant myocardial dysfunction [1]. Myocarditis occurring almost at the onset of bacterial gastroenteritis has been rarely reported in the literature, with Campylobacter spp. and Salmonella spp. being the main responsible bacteria. The lack of clinical suspicion and the high percentage of self-limited clinical course contribute to the underestimation of the true incidence of myocarditis in general.

In our literature review, we have included cases of Salmonella myocarditis, and we discuss current trends on the use of diagnostic tools employed to establish the diagnosis in these instances (Table 1) [2-15].

In the case presented, our patient was immunocompetent, and no bacteremia or sepsis could be established as possible explanation of myocardial involvement. It is worth mentioning that the duration of symptoms was only 30 minutes with complete resolution of chest pain, while usual biomarkers of cardiac injury (CK, AST, and LDH) were only 


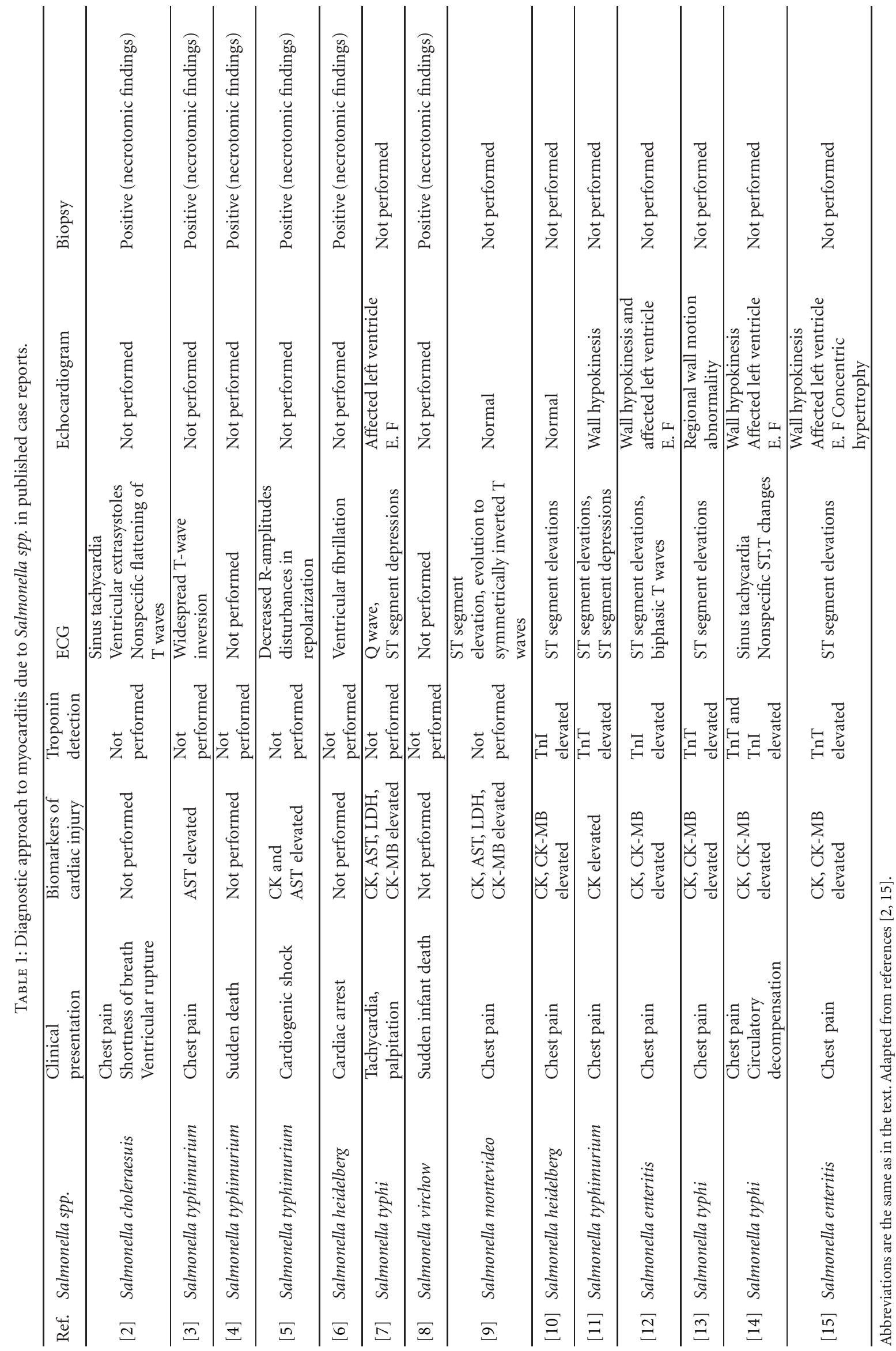




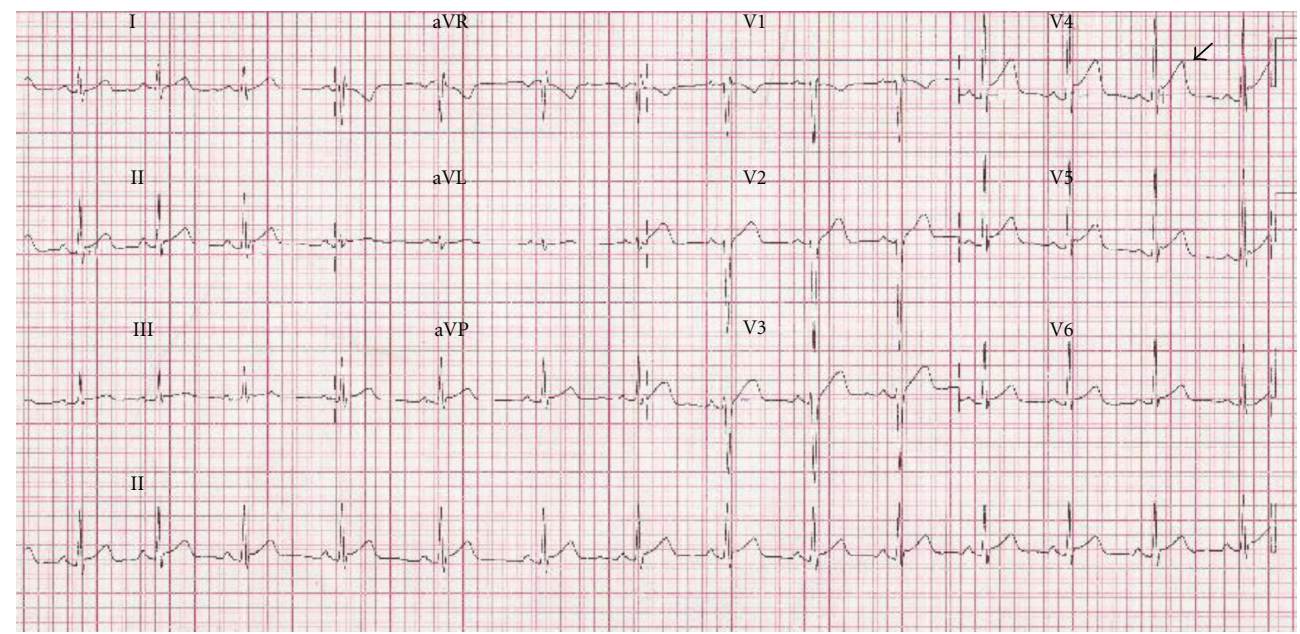

(a)

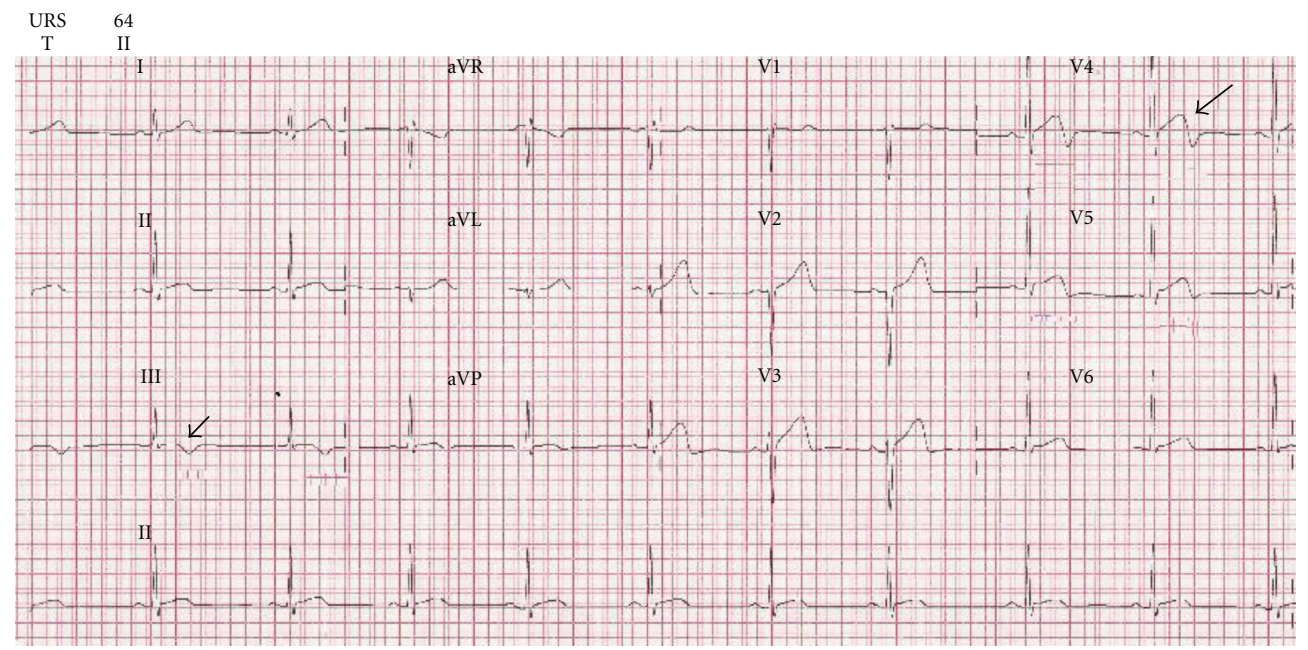

(b)

FIGURE 2: (a) ECG on admission, with changes due to early repolarization. (b) ECG on the third day, with T wave inversion in lead III and biphasic $T$ wave in leads $V_{4}$ and $V_{5}$.

mildly elevated. ECG had initially no pathological findings, and ECHO performed was repeatedly normal. These observations show that myocardial involvement might have been possibly left unrecognized without determination of cardiac troponin I.

There are six previous reports on the use of cardiac troponin in the diagnosis of Salmonella myocarditis [10-15], while the diagnostic value of this biomarker is well established in the literature for myocarditis [17, 18]. Cardiac troponin has limited sensitivity (34\%) for the diagnosis of myocarditis with a specificity reaching 89\% [18]. Still, compared to other biomarkers of cardiac injury, its sensitivity prevails (CK and CK-MB) for the detection of myocyte injury in myocarditis. We have always to keep in mind that cardiac troponin has low negative predictive value, indicating that normal values do not exclude myocardial injury. As indicated by our case and previous reports [10-15], the use of troponin is recommended in patients with gastroenteritis who complain of chest pain, regardless of the duration of symptoms.
ECG clearly remains a useful tool for the diagnosis of myocarditis. Even though findings exclusive for myocarditis, do not exist, serial ECG findings are characteristic and often extremely helpful for the distinction from acute myocardial infarction [1]. Certain ECG abnormalities like the presence of Q waves or left bundlebranch block are reported to have prognostic value $[19,20]$. In a study involving mostly cases with idiopathic myocarditis the presence of ECG abnormalities had low sensitivity (47\%) [19]. In our review of cases with Salmonella-associated myocarditis, ECG changes were present in all but 2 of them in which ECG was not performed (Table 1) [4, 8]. Whether differences amongst myocarditis of diverse etiologies exist in terms of ECG findings needs further investigation.

Echocardiography is valuable in the diagnostic evaluation of myocarditis though no specific findings exist and all patterns of cardiomyopathy have been described in this clinical entity $[1,21]$. ECHO can detect wall motion abnormalities, disturbances of ventricular function, whose presence has prognostic value, and can also distinguish 
fulminant from acute myocarditis [22]. Of relevance, with the use of ECHO, other causes of cardiac dysfunction can be excluded, serial monitoring of patients performed, and response to treatment assessed. Repeated ECHO evaluations in our patient was normal, as was also the case in other 2 previous reports (Table 1) $[9,10]$.

The application of novel imaging techniques and especially of contrast-enhanced magnetic resonance imaging (MRI) is very promising in the diagnostic assessment of patients with myocarditis [23]. The use of MRI should be considered especially in circumstances where both conventional imaging methods and other diagnostic tools fail to detect myocardial dysfunction.

Endomyocardial biopsy (EMB) still remains the gold standard for establishing the diagnosis of myocarditis with the use of Dallas criteria [24]. However, low sensitivity (ranging from $10 \%$ to $22 \%$ in various reports), sampling errors, variability in interpretation of samples, and lack of prognostic value have raised concerns on the diagnostic limitations of EMB [1,25]. Recently, recommendations regarding patients who will benefit from EMB and, therefore, should undergo this procedure have resolved the above mentioned issues [26]. New imaging techniques, especially MRI with performance of MRI-guided EMB, seem to be very promising considering the reduction of sampling errors during EMB and the increase in sensitivity [26]. Taking into account the self-limited course of myocarditis in our patient, we did not consider performing EMB. Review of the literature revealed no cases that had undergone EMB for Salmonella myocarditis. To our knowledge, positive EMB are reported only from necrotomies. These data reflect the above-mentioned difficulties, limitations of the procedure, and the increasing trend to perform EMB in selected group of patients.

\section{Conclusions}

Myocarditis after Salmonella spp. infection is rare though its real incidence remains unknown. As evolution to dilated cardiomyopathy and sudden death are known complications of myocarditis, its occurrence must be excluded in patients with gastroenteritis who complain of chest pain.

Our review of the current diagnostic approach of patients with possible myocarditis clearly shows the absence of a single marker or procedure to establish the diagnosis. Even though new assays and imaging techniques are extremely helpful and promising, it is the physician's diagnostic suspicion that will guide the diagnostic evaluation with the proper tools in order to rule out other causes of cardiac dysfunction and verify myocarditis.

\section{References}

[1] L. T. Cooper Jr., "Myocarditis," The New England Journal of Medicine, vol. 360, no. 15, pp. 1526-1538, 2009.

[2] V. Sanders and L. F. Misanik, "Salmonella myocarditis. Report of a case with ventricular rupture," American Heart Journal, vol. 68 , no. 5 , pp. 682-685, 1964.
[3] K. B. Shilkin, "Salmonella typhimurium pancarditis," Postgraduate Medical Journal, vol. 45, no. 519, pp. 40-43, 1969.

[4] J. Simonsen and E. Falk, "A case of sudden cardiac death in connection with Salmonella typhimurium infection," Forensic Science International, vol. 16, no. 3, pp. 283-287, 1980.

[5] M. Gotz and R. Juchems, "Myocarditis caused by Salmonella typhimurium," Klinische Wochenschrift, vol. 61, no. 22, pp. 1153-1157, 1983.

[6] C. R. Burt, J. C. Proudfoot, M. Roberts, and R. H. Horowitz, "Fatal myocarditis secondary to Salmonella septicemia in a young adult," Journal of Emergency Medicine, vol. 8, no. 3, pp. 295-297, 1990.

[7] H. Akdeniz, I. Tuncer, H. Irmak, and A. P. Demiröz, "Salmonella myocarditis in a patient with Wolf-ParkinsonWhite syndrome that was confused with an inferior myocardial infarction," Clinical Infectious Diseases, vol. 25, no. 3, pp. 736-737, 1997.

[8] C. Neuwirth, C. Francois, N. Laurent, and A. Pechinot, "Myocarditis due to Salmonella virchow and sudden infant death," The Lancet, vol. 354, no. 9183, p. 1004, 1999.

[9] K. O’Connor, "Acute myocarditis precipitated by Salmonella Montevideo infection: a case report," Irish Medical Journal, vol. 93, no. 1, pp. 21-22, 2000.

[10] P. Wanby and B. Olsen, "Myocarditis in a patient with salmonella and campylobacter enteritis," Scandinavian Journal of Infectious Diseases, vol. 33, no. 11, pp. 860-862, 2001.

[11] P. Williams and J. Lainchbury, "Enteritis-associated myocarditis," Heart Lung and Circulation, vol. 13, no. 1, pp. 106-109, 2004.

[12] P. Franczuk, K. Rewiuk, and T. Grodzicki, "Myocarditis related to Salmonella enteritidis infection," Cardiology Journal, vol. 14, no. 6, pp. 589-591, 2007.

[13] I. Sathyamurthy, P. R. Vidyalakshmi, and K. Jayanthi, "salmonella myocarditis presenting as acute myocardial infarction: a case report," Indian Heart Journal, vol. 60, no. 6, pp. 602-604, 2008.

[14] R. F. Al-Aqeedi, A. Kamha, F. K. Al-Aani, and A. A. Al-Ani, "Salmonella myocarditis in a young adult patient presenting with acute pulmonary edema, rhabdomyolysis, and multiorgan failure," Journal of Cardiology, vol. 54, no. 3, pp. 475479, 2009.

[15] B. Rossetti, G. Nguisseu, A. Buracci, L. Migliorini, and G. Zanelli, "Myocarditis mimicking an acute coronary syndrome: a case related to Salmonella enteritis," Gastroenterology Research and Practice, vol. 2009, Article ID 931853, 2 pages, 2009.

[16] G. M. Felker, W. Hu, J. M. Hare, R. H. Hruban, K. L. Baughman, and E. K. Kasper, "The spectrum of dilated cardiomyopathy. The Johns Hopkins experience with 1,278 patients," Medicine, vol. 78, no. 4, pp. 270-283, 1999.

[17] B. Lauer, C. Niederau, U. Kühl et al., "Cardiac troponin T in patients with clinically suspected myocarditis," Journal of the American College of Cardiology, vol. 30, no. 5, pp. 1354-1359, 1997.

[18] S. C. Smith, J. H. Ladenson, J. W. Mason, and A. S. Jaffe, "Elevations of cardiac troponin I associated with myocarditis. Experimental and clinical correlates," Circulation, vol. 95, no. 1, pp. 163-168, 1997.

[19] T. Morgera, A. Di Lenarda, L. Dreas et al., "Electrocardiography of myocarditis revisited: clinical and prognostic significance of electrocardiographic changes," American Heart Journal, vol. 124, no. 2, pp. 455-467, 1992.

[20] H. Nakashima, T. Katayama, M. Ishizaki, M. Takeno, Y. Honda, and K. Yano, "Q wave and non-Q wave myocarditis 
with special reference to clinical significance," Japanese Heart Journal, vol. 39, no. 6, pp. 763-774, 1998.

[21] B. Pinamonti, E. Alberti, A. Cigalotto et al., "Echocardiographic findings in myocarditis," American Journal of Cardiology, vol. 62, no. 4, pp. 285-291, 1988.

[22] G. M. Felker, J. P. Boehmer, R. H. Hruban et al., "Echocardiographic findings in fulminant and acute myocarditis," Journal of the American College of Cardiology, vol. 36, no. 1, pp. 227$232,2000$.

[23] H. N. Skouri, G. W. Dec, M. G. Friedrich, and L. T. Cooper, "Noninvasive imaging in myocarditis," Journal of the American College of Cardiology, vol. 48, no. 10, pp. 2085-2093, 2006.

[24] H. T. Aretz, M. E. Billingham, W. D. Edwards et al., "Myocarditis. A histopathologic definition and classification," The American Journal of Cardiovascular Pathology, vol. 1, no. 1, pp. 3-14, 1987.

[25] I. Kindermann, M. Kindermann, R. Kandolf et al., "Predictors of outcome in patients with suspected myocarditis," Circulation, vol. 118, no. 6, pp. 639-648, 2008.

[26] L. T. Cooper, K. L. Baughman, A. M. Feldman et al., “The role of endomyocardial biopsy in the management of cardiovascular disease: a scientific statement from the American Heart Association, the American College of Cardiology, and the European Society of Cardiology," Circulation, vol. 116, no. 19, pp. 2216-2233, 2007. 


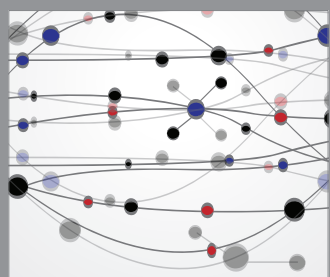

The Scientific World Journal
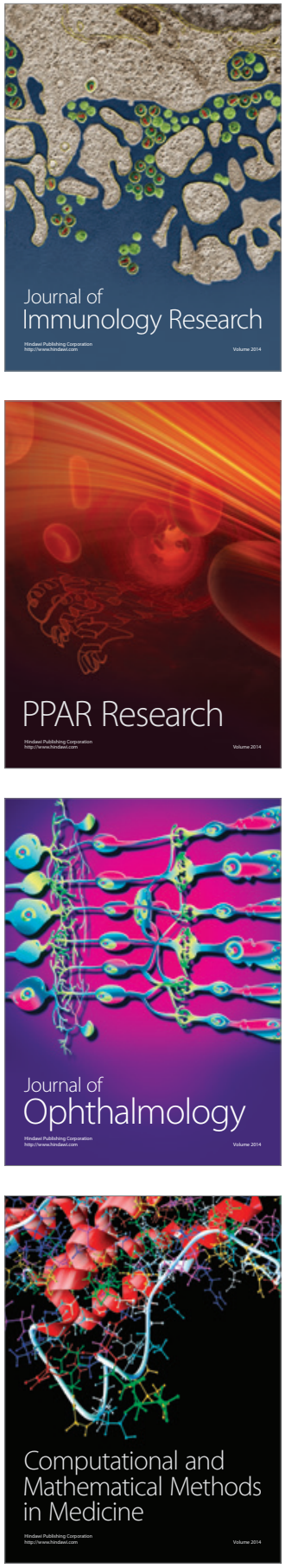

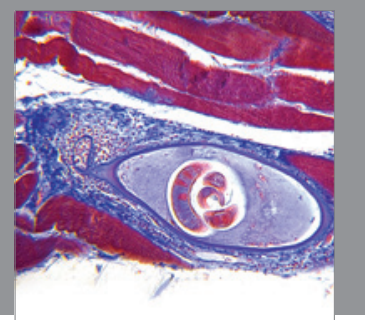

Gastroenterology

Research and Practice
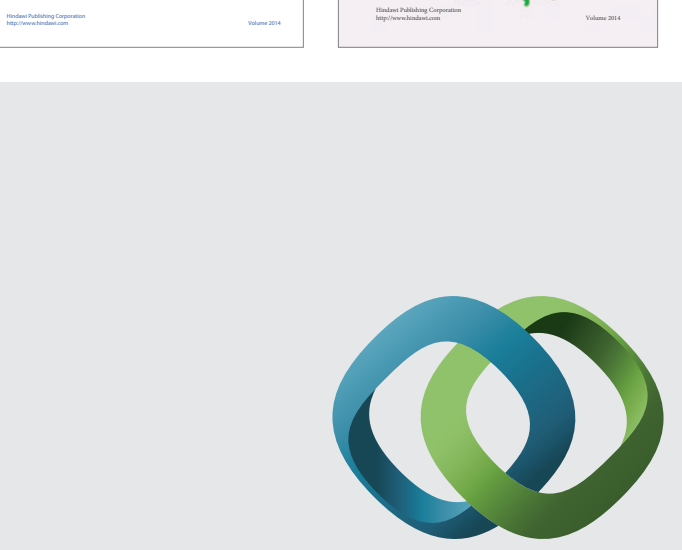

\section{Hindawi}

Submit your manuscripts at

http://www.hindawi.com
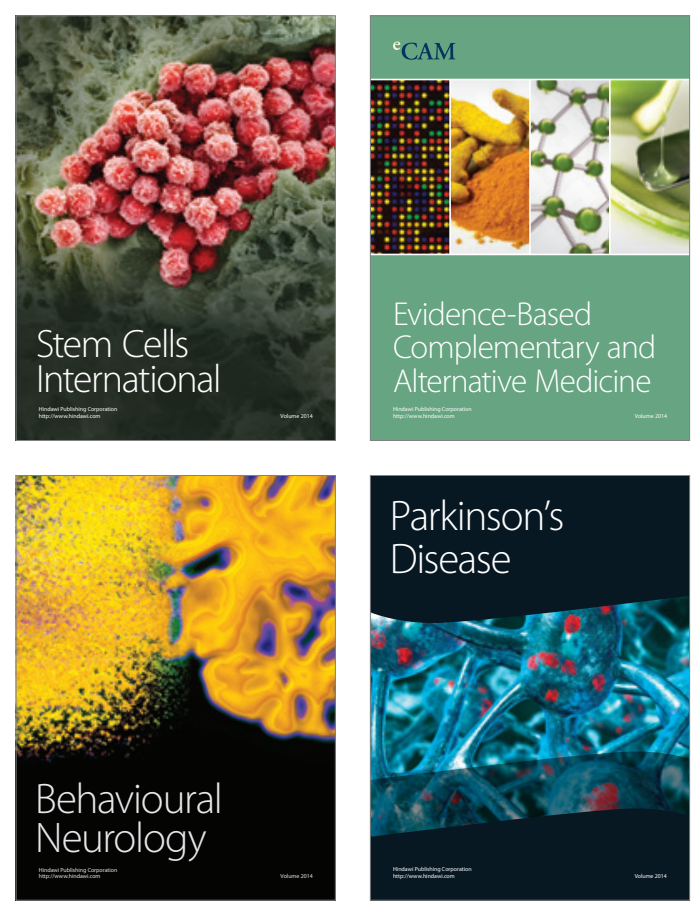

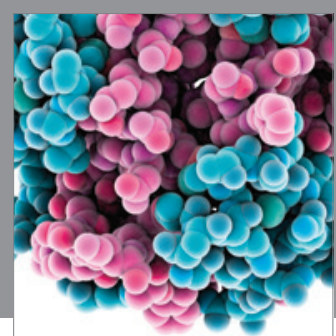

Journal of
Diabetes Research

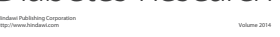

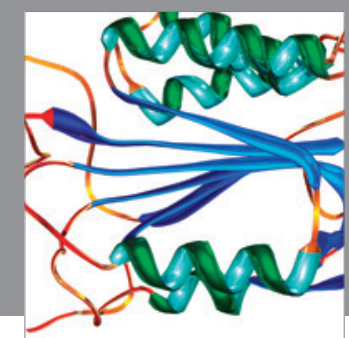

Disease Markers
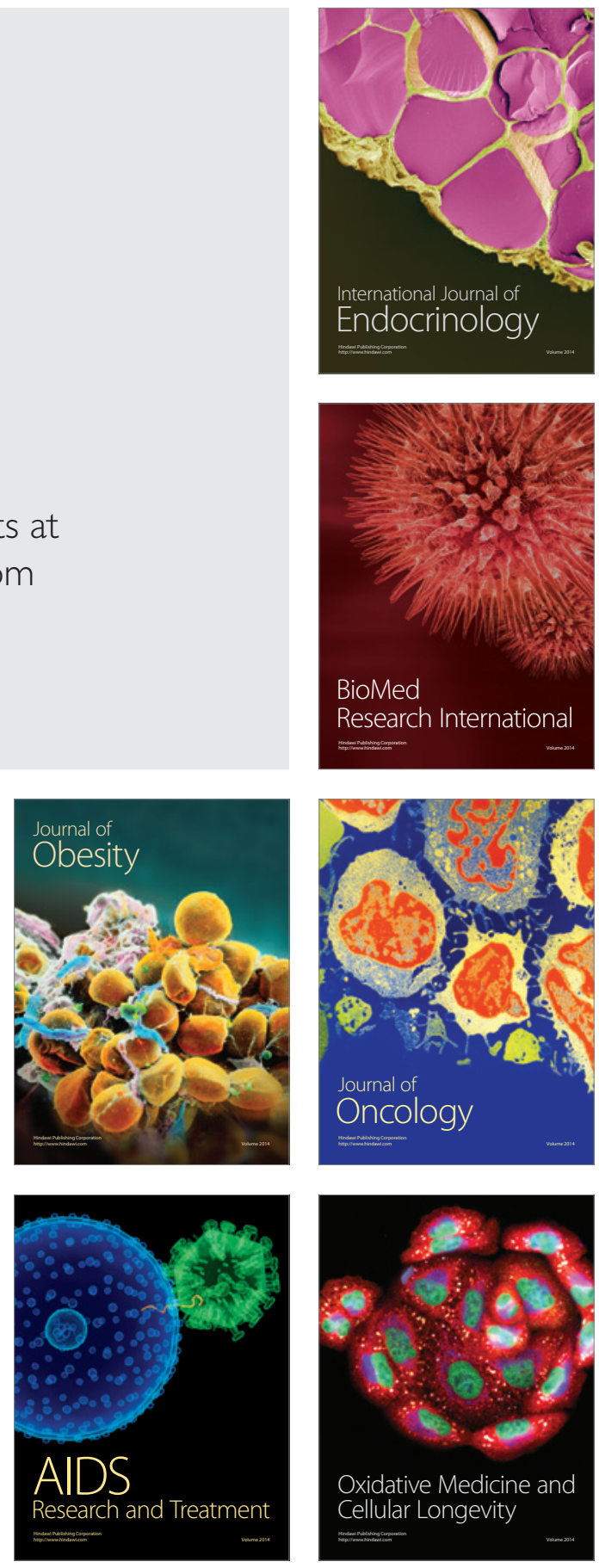